\title{
HDL from CETP-deficient subjects shows enhanced ability to promote cholesterol efflux from macrophages in an apoE- and ABCG1-dependent pathway
}

\author{
Fumihiko Matsuura, ${ }^{1}$ Nan Wang, ${ }^{1}$ Wengen Chen, ${ }^{1}$ Xian-Cheng Jiang, ${ }^{2}$ and Alan R. Tall1
}

1Division of Molecular Medicine, Department of Medicine, Columbia University, New York, New York, USA. 2Department of Anatomy and Cell Biology and Scientific Computing Center, State University of New York, Downstate Medical Center, New York, New York, USA.

\begin{abstract}
Genetic deficiency or inhibition of cholesteryl ester transfer protein (CETP) leads to a marked increase in plasma levels of large HDL-2 particles. However, there is concern that such particles may be dysfunctional in terms of their ability to promote cholesterol efflux from macrophages. Recently, the ATP-binding cassette transporter ABCG1, a macrophage liver $X$ receptor (LXR) target, has been shown to stimulate cholesterol efflux to HDL. We have assessed the ability of HDL from subjects with homozygous deficiency of CETP (CETP-D) to promote cholesterol efflux from macrophages and have evaluated the role of ABCG1 and other factors in this process. CETP-D HDL-2 caused a 2- to 3-fold stimulation of net cholesterol efflux compared with control HDL-2 in LXR-activated macrophages, due primarily to an increase in lecithin:cholesterol acyltransferase-mediated (LCAT-mediated) cholesteryl ester formation in media. Genetic knockdown or overexpression of ABCG1 showed that increased cholesterol efflux to CETP-D HDL was ABCG1 dependent. LCAT and apoE contents of CETP-D HDL-2 were markedly increased compared with control HDL-2, and increased cholesterol esterification activity resided within the apoE-HDL fraction. Thus, CETP-D HDL has enhanced ability to promote cholesterol efflux from foam cells in an ABCG1-dependent pathway due to an increased content of LCAT and apoE.
\end{abstract}

\section{Introduction}

Plasma HDL levels are inversely related to the risk of atherosclerotic cardiovascular disease. Glomset first proposed that HDL might mediate the transport of cholesterol from peripheral tissues back to the liver (1). Central to this hypothesis was the concept that the lecithin:cholesterol acyltransferase (LCAT) enzyme in HDL would convert free cholesterol (FC) to cholesteryl ester (CE), thereby creating a gradient for FC efflux from cells to HDL. Cholesterol exchange between cells and HDL can occur by aqueous diffusion and may be facilitated by scavenger receptor, class B, type I (SR-BI) (2). However, these pathways do not result in net cholesterol efflux unless accompanied by a compositional change in HDL, such as that produced by the LCAT reaction. ABC transporters can promote net movement of cholesterol from cells to acceptors in medium. ABCA1, the defective gene in Tangier disease, promotes net phospholipid and cholesterol efflux from cells to lipid-poor apoA-I, decreasing cholesterol accumulation in macrophage foam cells and initiating HDL formation in the liver (3). However, ABCA1 interacts poorly with plasma HDL (4). Recently, we and others have shown that another transporter, ABCG1, promotes mass cholesterol efflux from cells

Nonstandard abbreviations used: AcLDL, acetyl-LDL; CE, cholesteryl ester; CETP, CE transfer protein; CETP-D, CETP deficiency, CETP-deficient; E600, diethyl p-nitrophenyl phosphate; FC, free cholesterol; HEK, human embryonic kidney; LCAT, lecithin:cholesterol acyltransferase; LXR, liver X receptor; PC, phosphatidylcholine; SM, sphingomyelin; SMase, sphingomyelinase; SR-BI, scavenger receptor, class B, type I; T0, T0901317; TC, total cholesterol; TG, triglyceride.

Conflict of interest: Alan R. Tall reports being a consultant to Pfizer, Merck, and Abbott Pharmaceuticals.

Citation for this article: J. Clin. Invest. 116:1435-1442 (2006). doi:10.1172/JCI27602. to mature HDL particles, but not to lipid-poor apoA-I $(5,6)$. Both ABCA1 and ABCG1 are abundant in macrophages, especially following cholesterol loading and activation of the transcription factors liver X receptor/retinoid X receptor (LXR/RXR), which target the promoters of both transporters $(7,8)$.

In humans, CE generated by LCAT in HDL is transferred to other lipoproteins by CE transfer protein (CETP). CETP promotes the removal of CE from HDL in exchange for triglycerides (TGs) derived primarily from very low-density lipoprotein (VLDL) or chylomicrons. Homozygous genetic deficiency of CETP due to an intron 14 splicing defect results in absence of CETP in plasma, dramatic elevations in HDL cholesterol and apoA-I levels, and moderate reductions in LDL cholesterol and apoB levels (9). Based on this phenotype, CETP inhibition was proposed as a strategy to increase HDL levels in humans $(10,11)$. Potent CETP inhibitors have been developed that cause marked increases in HDL levels and smaller decreases in LDL cholesterol levels (12-14).

Despite the dramatic lipoprotein phenotype, there has been concern that large CE-rich HDL particles accumulating in CETP deficiency (CETP-D) might have impaired ability to mediate cholesterol efflux from macrophage foam cells (15). In part, the concern is related to the fact that ABCA1 only uses small pre- $\beta$ HDL particles or lipid-poor apoA-I as a substrate, and such particles are not increased in CETP-D (16). Indeed, since the hydrolysis of HDL TGs secondary to CETP-mediated CE-TG exchange results in the release of lipid-poor apoA-I, it is possible that the regeneration of apolipoprotein acceptors for ABCA1 is impaired in CETP-D. Moreover, in 1 study, CETP-D HDL showed decreased cholesterol efflux from foam cells compared with control HDL (17). The recent dis- 
A

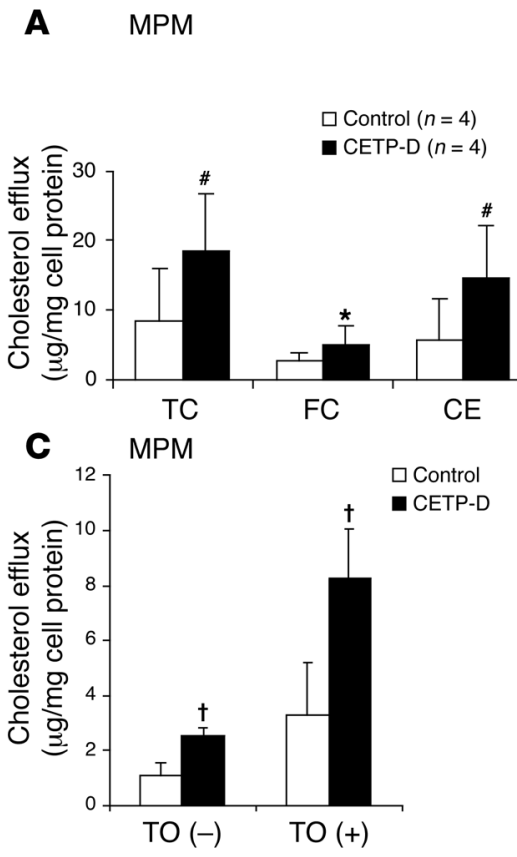

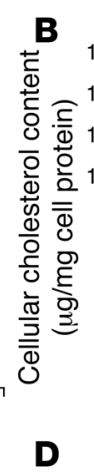

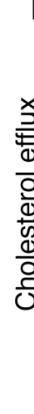

MPM

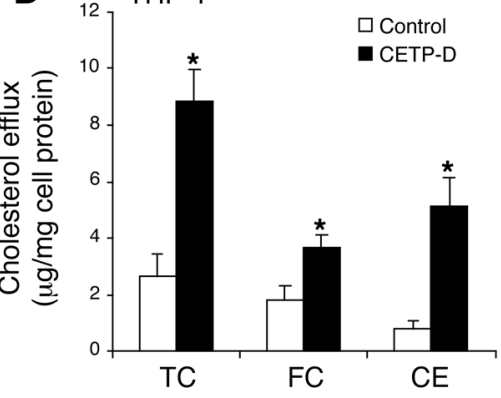

\section{Figure 1}

HDL-2-mediated mass cholesterol efflux from macrophages. Mouse peritoneal macrophages (MPMs) or THP- 1 cells were incubated for 24 hours in DMEM containing AcLDL $(50 \mu \mathrm{g}$ protein $/ \mathrm{ml})$ with T0 $(3 \mu \mathrm{M})$, then cholesterol efflux was performed for 8 hours with HDL (50 $\mu \mathrm{g} / \mathrm{ml}$ HDL protein) added to media. (A) HDL-2mediated cholesterol efflux from MPMs. The data show the increase in TC, FC, and CE mass in media and represent mean \pm SEM of 9 independent experiments. ${ }^{*} P<0.01,{ }^{\#} P<0.05$ versus control HDL-2. (B) Cholesterol removal from MPMs by HDL-2. The data show the mass of cholesterol remaining in cells at the end of the efflux period and represent mean \pm SEM of values from 9 independent experiments. ${ }^{*} P<0.01$, ${ }^{\#} P<0.05$ versus no addition of HDL-2; ${ }^{\dagger} P<0.05$ versus control HDL-2. (C) HDL-2mediated cholesterol efflux from MPMs treated with or without TO. The data show the increase in TC mass in media and represent mean \pm SD of an experiment performed in triplicate. ${ }^{*} P<0.05$, versus control HDL-2. (D) HDL-2-mediated cholesterol efflux from THP-1 macrophages. The data represent mean \pm SD of an experiment performed in triplicate. ${ }^{\star} P<0.01$, versus control HDL-2. covery of the role of ABCG1 in mediating cholesterol efflux to HDL particles, including HDL-2 $(5,6)$, has led us to reevaluate the ability of HDL from CETP-D subjects to mediate cholesterol efflux from macrophage foam cells and the role of ABCG1 in this process.

\section{Results}

Plasma lipoprotein characterization. The serum lipoprotein profile of 4 homozygous CETP-D patients is shown in Supplemental Table 1 (supplemental material available online with this article; doi:10.1172/JCI27602DS1). The serum HDL cholesterol levels in the patients were 3- to 4-fold higher compared with those in controls. The serum concentrations of apoA-I and apoE in the patients were also markedly increased. The HDL-2 from the patients $(n=4)$ was rich in CE and poor in TGs compared with controls (CE: $0.52 \pm 0.04$ [mean $\pm \mathrm{SD}$ ] versus $0.29 \pm 0.11 \mathrm{mg} / \mathrm{mg}$ HDL protein, $P=0.02$; TGs: $0.06 \pm 0.01$ versus $0.13 \pm 0.01 \mathrm{mg} / \mathrm{mg}$ HDL protein, $P=0.002$ ) (Supplemental Figure 1). However, there was no significant difference in $\mathrm{FC} /$ protein or total phospholipid/protein ratios or in HDL-3 composition when CETP-D and control subjects were compared. As assessed by the $4-15 \%$ nondenaturing PAGE, the particle size of CETP-D HDL-2 was substantially larger than that of control HDL-2 $(11.5 \pm 0.2$ and $9.7 \pm 0.1 \mathrm{~nm}[$ mean $\pm \mathrm{SD}], P<0.001)$. Thus, the compositional and size characteristics of HDL used in this study were similar to those in earlier reports (17-19).

CETP-D HDL-2 has enhanced ability to promote cholesterol efflux from macrophages, reflecting an increase in CE formation in media. ABCA1 and $\mathrm{ABCG} 1$ are induced in macrophage foam cells as a result of increased transcription mediated by $\operatorname{LXR} / \operatorname{RXR}(7,8)$. Therefore, we first investigated net cholesterol efflux to the HDL-2 from the CETP-D patients using mouse peritoneal macrophages treated with the LXR activator (T0901317 [T0]). In order to assess functionality of HDL particles, CETP-D and control HDL were added to media at the same protein concentration $(50 \mu \mathrm{g} / \mathrm{ml})$. As shown in Figure 1A, total net cholesterol efflux was increased 2.2-fold $(n=4 ; P=0.012)$ for CETP-D HDL, compared with control. This reflected a 2.5 -fold increase in $\mathrm{CE}$ accumulation in media and a 1.9-fold increase in FC. Conversely, measurement of cellular cholesterol mass showed a 2.6-fold-larger reduction in total cholesterol (TC) for CETP-D HDL-2 compared with control (Figure 1B), reflecting a larger decrease in cellular $\mathrm{CE}$ content. The percentage of TC efflux from cells was also significantly enhanced for CETP-D HDL-2, compared with control $(9.3 \% \pm 2.9 \%$ versus $3.6 \% \pm 2.1 \%$, respectively; $P=0.002)$. Thus, CETP-D HDL-2 had increased ability to decrease $\mathrm{CE}$ accumulation in macrophages.

To investigate the effect of LXR activation on HDL-2-mediated cholesterol efflux, we examined net TC efflux to HDL-2 from mouse macrophages treated with or without T0 (Figure 1C). LXR activation induced a 2- to 3-fold increase in mass cholesterol efflux. CETP-D HDL showed increased ability to promote cholesterol efflux for both basal and LXR-activated macrophages. Finally, CETP-D HDL-2 also had markedly enhanced ability to promote net cholesterol efflux from human THP-1 macrophages (Figure 1D). We also tested net cholesterol efflux from T0-stimulated THP-1 macrophages using 3 different concentrations of HDL-2 (Supplemental Figure 2). Increasing concentrations of HDL-2 enhanced net cholesterol efflux to both CETP-D and control HDL-2. The difference between CETP-D and control HDL-2 tended to increase at higher HDL concentration. These data suggest that in CETP-D, both a higher HDL concentration and increased cholesterol efflux potency of HDL particles could increase the cholesterol efflux potential of the HDL fraction. We next compared net cholesterol efflux to HDL-2 with that to HDL-3 in T0-stimulated mouse macrophages (Supplemental Figure 3). HDL-3 had less ability to promote net cholesterol efflux than HDL-2 and did not promote CE accumulation in media. Moreover, there was no significant difference in the cholesterol efflux between the CETP-D and the control HDL-3. This is consistent with the finding that CETP-D HDL-3, though slightly increased in amount, has similar composition and size to control HDL-3.

Effect of suppression of ABCG1 expression by siRNA on HDL-2-mediated cholesterol removal from mouse macrophages. Next, we examined the effect of suppression of ABCG1 by siRNA on HDL-2-mediated cho- 


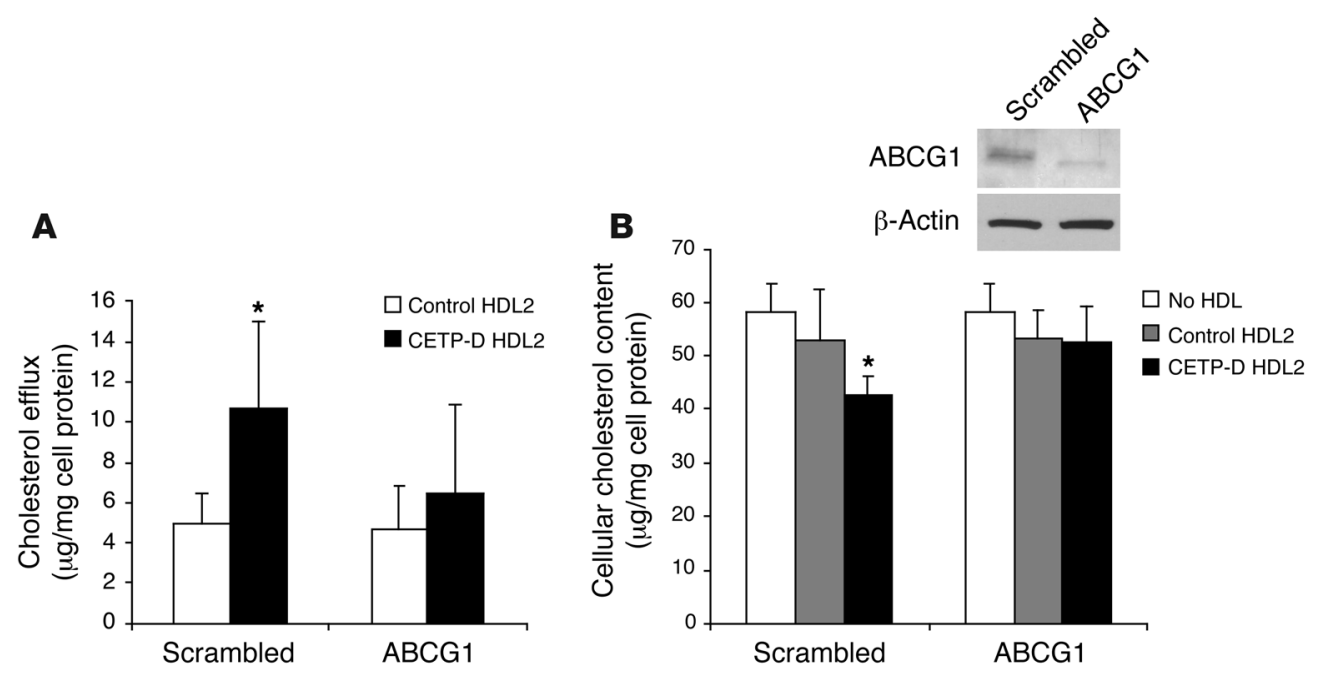

Figure 2

Effect of suppression of ABCG1 expression by siRNA on HDL-2-mediated cholesterol efflux from macrophages. Mouse macrophages (MPMs) were transfected with siRNA against ABCG1 or scrambled control siRNA (160 nM) for 48 hours. Cholesterol efflux was performed for 8 hours in DMEM containing HDL-2 (50 $\mathrm{gg} / \mathrm{ml} \mathrm{HDL}$ protein). (A) HDL-2-mediated cholesterol efflux from cells. The data show the increase in TC mass in media and represent mean $\pm \mathrm{SD}$ of an experiment performed in triplicate. ${ }^{*} P<0.05$ versus control HDL-2, in cells transfected with scrambled control siRNA. (B) The cholesterol removal from cells by HDL-2 was estimated by changed intracellular cholesterol mass in the cells. The data show TC mass remaining in cells at the end of the incubation period and represent mean \pm SD of an experiment performed in triplicate. ${ }^{*} P<0.05$ versus cells transfected with scrambled control siRNA. Protein level of ABCG1 normalized against $\beta$-actin was determined by Western blot analysis (inset).

lesterol efflux from T0-stimulated mouse macrophages (Figure 2). The removal of cholesterol by the CETP-D HDL-2 was significantly higher than that by the control HDL-2, as reflected by both the media and cellular cholesterol contents. The difference in cholesterol efflux between the CETP-D and the control HDL- 2 was abolished by the suppression of ABCG1 expression (ABCG1 protein expression in the macrophages transfected with siRNA for ABCG1 was $20-30 \%$ of that in the cells with scrambled control siRNA; Figure 2B, inset).

ABCG1-mediated cholesterol efflux from buman embryonic kidney 293 cells to HDL-2. To confirm that lipid-rich large HDL-2 has increased potency in promoting cholesterol efflux facilitated by ABCG1, we studied net cholesterol efflux to HDL-2 from ABCG1-transfected human embryonic kidney (HEK) 293 cells (Supplemental Figure 4). The ABCG1-mediated cholesterol efflux to the CETP-D HDL-2 from ABCG1-HEK 293 cells was significantly increased compared with that from the control. The increased cholesterol efflux to the CETP-D HDL-2 was due to enhanced accumulation of both CE and FC. Together these results indicate that CETP-D HDL-2 enhances cellular cholesterol efflux in an ABCG1-dependent fashion.

Isotopic cholesterol efflux to HDL from T0-stimulated mouse macrophages. The isotopic cholesterol efflux to the CETP-D HDL-2 was slightly but significantly increased compared with that of the control HDL-2 (Supplemental Figure 5), while the HDL-3 fractions showed no difference. While these data could suggest an increase in net cholesterol efflux for CETP-D HDL, isotopic efflux data are difficult to interpret because of the large contribution of cholesterol exchange.

Immunoblot analysis of LCAT, apoA-I, and apoE in HDL-2. The prominent increase in CE mass in media suggested an increase in LCATmediated cholesterol esterification in CETP-D HDL in the pres- ence of cells $(20,21)$. Thus, we next evaluated the presence of the LCAT enzyme in HDL by Western blotting. The same amount of total protein was analyzed for each sample. Since apoA-I is the main protein of HDL, the level of apoA-I appeared similar for control and CETP-D HDL. Interestingly, LCAT mass in CETP-D HDL-2 was markedly increased (mean 9.4-fold) compared with the control (Figure 3A). Furthermore, the content of apoE, a potential LCAT activation factor in larger lipoprotein particles (22), was also increased (mean 4.5-fold), as described previously (19).

To directly evaluate whether there was increased CE formation in media, cells were labeled with $\left[{ }^{3} \mathrm{H}\right]$ acetyl-LDL $\left(\left[{ }^{3} \mathrm{H}\right] \mathrm{AcLDL}\right)$ and washed extensively, HDL was added to media, and formation of $\left[{ }^{3} \mathrm{H}\right] \mathrm{CE}$ in media was determined. This showed an increase in $\left[{ }^{3} \mathrm{H}\right] \mathrm{CE}$ formation in media containing CETP-D HDL (Figure 3B). Moreover, this increase was markedly reduced by preincubation of HDL with an LCAT inhibitor (diethyl $p$-nitrophenyl phosphate [E600]). This effect was incomplete, likely due to partial reversal of LCAT inhibition when the inhibitor was diluted into media for the efflux experiments. To further evaluate the role of LCAT in cholesterol efflux activity, the mass efflux was measured in the presence of E600 (Figure 3C). The inhibitor markedly reduced total and CE accumulation in media. Thus, the increase in total and esterified cholesterol in media is primarily due to increased LCAT-mediated cholesterol esterification. However, in the presence of the E600, the CETP-D HDL still showed an increase in FC efflux compared with control, indicating that CETP-D HDL has an LCAT-independent ability to promote higher levels of FC efflux (Figure 3C, middle panel), indicating an additional property of the CETP-D HDL-2 that increases cholesterol efflux potential.

Relationship between sphingomyelin content of HDL-2 and cellular cholesterol efflux. Recently, Bolin et al. reported that sphingomyelin (SM) inhibits the binding of LCAT to HDL (23). Furthermore, Subbaiah et al. showed that a decreased SM/phosphatidylcholine (SM/PC) ratio of HDL-2 after treatment with sphingomyelinase (SMase) increases LCAT activity (24). This led us to measure the PC/SM ratio in the HDL-2 fractions (Figure 4). The SM/PC ratio of HDL-2 from the CETP-D patients was significantly decreased compared with that of the control HDL-2 $(0.10 \pm 0.02$ versus $0.19 \pm 0.01$, respectively; $P<0.005)$. However, there was no significant difference in the SM/PC ratio between the CETP-D and the control HDL-3.

To further explore a potential relationship between the SM/PC ratio of HDL and cholesterol efflux, we pretreated HDL-2 with SMase and then carried out cholesterol efflux studies. The SM/PC 
A

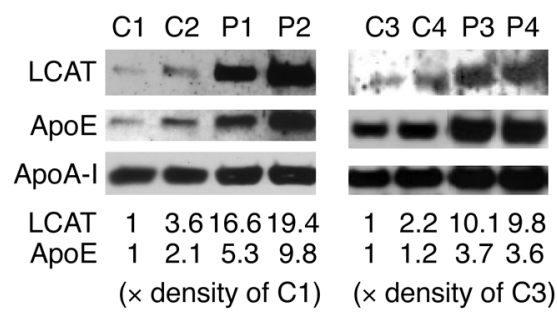

B

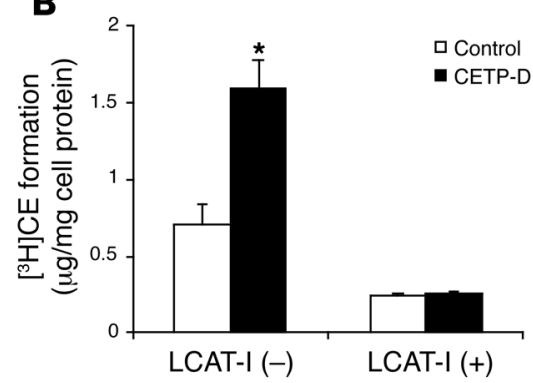

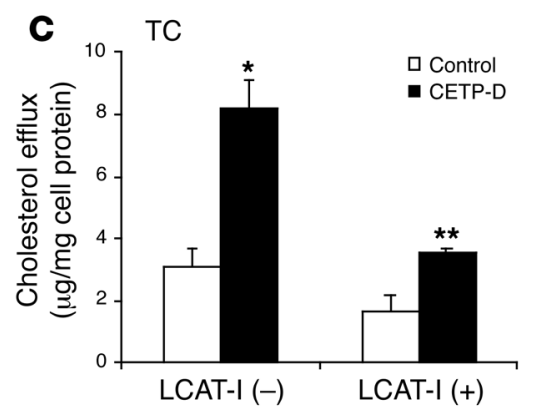
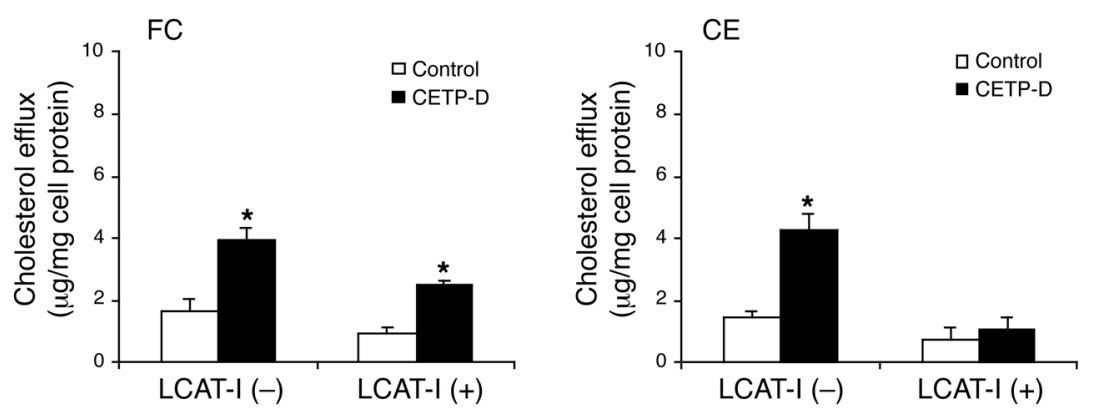

Figure 3

Analysis of LCAT, apoA-I, and apoE mass in HDL-2, and the effect of LCAT inhibitor on net cholesterol efflux to HDL-2 from macrophages and isotopic CE formation in media containing HDL-2. (A) Twenty micrograms HDL protein was analyzed by SDS-PAGE, blotted onto a nitrocellulose membrane, and incubated with indicated antibodies. C1-4, control HDL-2; P1-P4, CETP-D HDL-2. (B) Isotopic CE formation in media containing HDL-2. THP-1 macrophages were incubated in RPMI-1640 containing [ $\left.{ }^{3} \mathrm{H}\right]$ AcLDL with T0 for 24 hours, then cholesterol efflux was performed for 8 hours in RPMI-1640 containing HDL-2 $(50 \mu \mathrm{g} / \mathrm{ml}$ HDL protein) preincubated with or without 2 mM of LCAT inhibitor (LCAT-I; E600). The data represent mean $\pm \mathrm{SD}$ of an experiment performed in triplicate. ${ }^{*} P<0.005$, versus control HDL-2. (C) Net cholesterol efflux to HDL-2 treated with E600 from THP-1 macrophages. Macrophages were treated with T0 and AcLDL for 24 hours, then cholesterol efflux was performed for 8 hours in RPMI-1640 containing HDL-2 (50 $\mathrm{g} / \mathrm{ml} \mathrm{HDL}$ protein) pretreated with or without E600. The data represent mean \pm SD of an experiment performed in triplicate. ${ }^{\star} P<0.005,{ }^{\star \star} P<0.0005$, versus control HDL-2. Similar data were obtained in 2 separate experiments.

ratio of normal HDL-2 treated with SMase was decreased to about $60 \%$ of that of nontreated normal control HDL-2 (0.19 \pm 0.01 versus 0.12 , respectively) (Figure 4). Compared with nontreated control HDL-2, the SMase-treated normal HDL-2 showed enhanced cholesterol efflux, as indicated by an increase in both FC and CE in media (Figure 4). However, in this instance, the efflux was primarily driven by $\mathrm{FC}$ rather than $\mathrm{CE}$ formation.

The role of apoE in cholesterol efflux to HDL-2 in T0-stimulated macrophages. The ability of HDL from homozygous CETP-D patients to promote cholesterol efflux from mouse macrophages was previously investigated by Ishigami et al. (17), who showed that apoEfree HDL-2 in CETP-D could not protect macrophages from cholesterol accumulation. In that study, apoE was removed from the HDL fraction by heparin affinity chromatography. Therefore, we investigated whether the difference from the results of the earlier study could be related to the removal of apoE from the samples. We used heparin-sepharose affinity chromatography to isolate apoE-free HDL-2 from the holo-HDL-2 fraction. In this experiment, the holo-HDL-2 from a CETP-D subject mediated increased net cholesterol efflux and CE formation from macrophages, as expected. However, the apoE-free HDL-2 had diminished ability to promote cholesterol efflux, and there was no difference between CETP-D and control HDL-2 (Figure 5, B and $\mathrm{C}$ ). Interestingly, there was no increase in CE in media for apoE-free HDL-2, even though this fraction still contained LCAT (Figure 5A). This suggests that LCAT activation in the HDL-2 fraction depends on apoE. Consistent with this idea, the abil- ity of CETP-D HDL-2 to promote CE accumulation in media required the presence of apoE (Figure $5 \mathrm{C}$ ). Moreover, the apoEcontaining fraction of CETP-D HDL-2 showed enhanced cholesterol esterification activity (as shown by a drop in FC content) when incubated in a cell-free system, while the apoE-free HDL-2 fraction showed very little FC-to-CE conversion for either control or CETP-D HDL (Figure 5D). Of the total HDL-2 fraction, there was more cholesterol in the apoE-containing fraction for the CETP-D sample (25.6\% of total HDL-2 cholesterol), compared with the control (17.4\% of total HDL- 2 cholesterol). Thus, the substantially increased cholesterol esterification and cholesterol efflux activities of CETP-D HDL-2 reside in a somewhat more abundant apoE-containing fraction.

In view of the increased content of apoE in CETP-D HDL, we also considered the possibility that there might be a change in binding or particle reuptake of HDL-2 by cells. Indeed, we found that specific binding of CETP-D HDL-2 to cells was slightly increased and that a larger component of binding was inhibited by heparin for CETP-D HDL (Supplemental Figure 6). Thus, we considered the possibility that increased net cholesterol efflux might be related to increased apoE-mediated particle binding to cells. However, we found that heparin treatment actually led to an increase in cholesterol accumulation in media that was similar for control and CETP-D HDL (Supplemental Figure 6), most likely reflecting decreased apoE-mediated particle uptake. This excludes the possibility that differences in $\mathrm{CE}$ accumulation in media are related to a role of apoE in binding or reuptake of particles. 

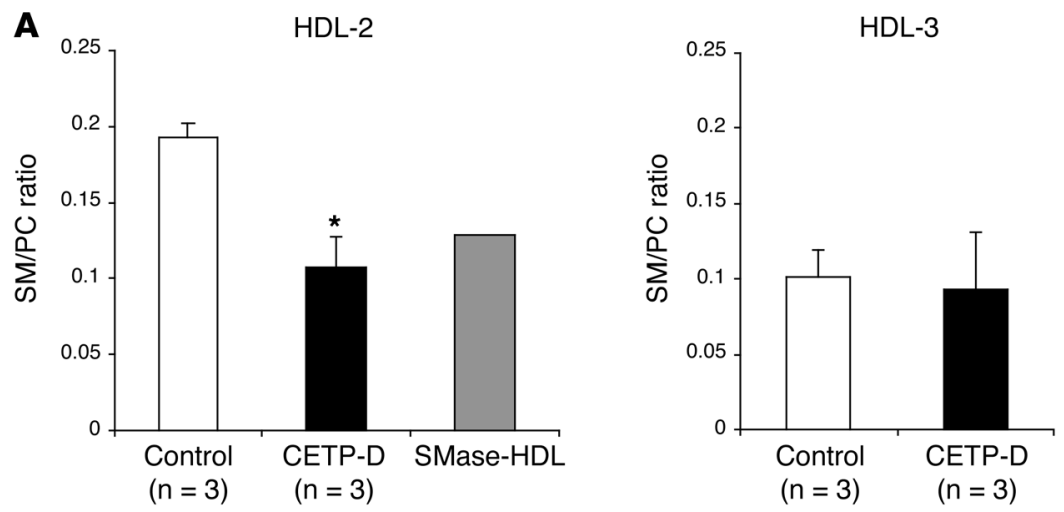

B

Figure 4

Analysis of SM mass in HDL-2 and HDL-3 and SMase-treated HDL-2-mediated cholesterol efflux from macrophages. (A) SM/PC ratio of HDL-2 and HDL-3. The incubation of normal HDL-2 with SMase was carried out for 1 hour at $37^{\circ} \mathrm{C}$ using $1.4 \mathrm{U}$ SMase/mg HDL protein. The data represent mean $\pm \mathrm{SD}$. ${ }^{*} P<0.05$, versus control HDL-2. (B) SMase-treated HDL-2-mediated cholesterol efflux from T0-stimulated mouse macrophages. Macrophages were treated with T0 $(3 \mu \mathrm{M})$ and with AcLDL $(50 \mu \mathrm{g}$ protein/ml) for 24 hours, and then cholesterol efflux was performed for 8 hours in DMEM containing HDL-2 (50 $\mu \mathrm{g} / \mathrm{ml} \mathrm{HDL}$ protein). The data represent mean \pm SD of an experiment performed in triplicate. $\# P<0.01$, versus normal control HDL-2. Similar data were obtained in 2 separate experiments.

\section{Discussion}

We undertook this study in order to evaluate the functionality of HDL from subjects with genetic CETP-D in terms of its ability to promote cholesterol efflux from macrophages. Surprisingly, we found enhanced cholesterol efflux for CETP-D HDL. This appears to be related to a very high content of LCAT and apoE in HDL particles from CETP-D subjects, driving net cholesterol efflux by promoting cholesterol esterification in the HDL particles. Our studies reveal a key role of LCAT and apoE in mass cholesterol efflux and show that large HDL can directly mediate cholesterol efflux in an ABCG1-dependent fashion. Our data are consistent with earlier studies, in which Mahley and associates showed that large, apoE-rich HDL decreased $\beta$-VLDL-induced cholesterol and CE accumulation in foam cells in association with protection from atherosclerosis in different animals models (25). Our mechanistic findings also match the extensive epidemiological data indicating that subjects with high levels of large-sized HDL particles $(\alpha-1, \alpha-2$, pre- $\alpha-1$, and pre- $\alpha-2)$ or specifically the HDL-2 ( $\alpha-1$ and $\alpha-2)$ subclass may be protected from atherosclerotic cardiovascular disease (26).

The finding of high LCAT protein levels in CETP-D HDL might be related to the decrease in SM/PC ratio in CETP-D HDL particles. Alternatively it could be related to increased particle size or $\mathrm{CE}$ content. In reconstituted HDL particles, an increasing ratio of $\mathrm{SM} / \mathrm{PC}$ was shown to inhibit the LCAT reaction, by decreasing the binding of enzyme to the HDL (23). Thus, for a constant amount of LCAT, decreasing the SM/PC ratio of HDL would be expected to increase LCAT activity and cholesterol efflux, as observed following SMase treatment (Figure 4). In vivo it is possible that there is an equilibrium between LCAT bound to HDL and free LCAT and that catabolism occurs more rapidly from the non-HDL-bound pool; a similar situation has been described for CETP (27). The dramatic increase in LCAT protein observed in CETP-D HDL might reflect such a process in vivo. In the cell culture experiments, increases in both LCAT protein and activity appear to drive CE formation in media. Experiments using an LCAT inhibitor showed that the increase in cholesterol esterification activity was the primary mechanism driving cellular cholesterol efflux and CE formation in media (Figure 3). However, there was an additional component of efflux of FC that was independent of LCAT and increased in the CETP-D HDL. In part this could also be related to an altered SM/PC ratio of CETP-D HDL-2, since SMase treatment of normal HDL-2 results in a marked increase in FC efflux activity. There could also be other factors, such as an increase in apoA-I/apoA-II ratio that occurs in CETP-D HDL-2, as in some studies, lipoprotein apoA-I (LpA-I) has shown increased cholesterol efflux capacity compared with LpA-I/A-II (28).

Normally LDL is relatively enriched in SM compared with HDL. The decrease in SM/PC ratio observed in CETP-D HDL may arise because of a defect in exchange of $\mathrm{PC}$ and SM in vivo that would normally drive the SM/PC ratio of LDL and HDL toward equilibrium. In addition to its neutral lipid exchange activity, CETP promotes exchange of choline-containing phospholipids (PC, SM) between HDL and LDL (29). A related but functionally distinct plasma phospholipid transfer protein (PLTP) mediates the net transfer of phospholipids from TG-rich lipoprotein into HDL (30) but appears to be relatively inactive in the exchange of phospholipids between LDL and HDL (29). These distinct biochemical properties of CETP and PLTP may account for the changes in SM/PC ratio of CETP-D HDL. The decreased SM content of CETP-D HDL could also be a factor in increasing apoE in these particles, as SM has been shown to decrease binding of apoE to HDL (23).

A surprising finding was the apparent key role of apoE in stimulating the LCAT reaction within the HDL-2 fraction. Removal of the apoE from the HDL-2 by heparin affinity chromatography resulted in loss of the ability of HDL to promote CE accumulation in media and accounted for the difference in results between the present and an earlier study (17). Although apoA-I is able to activate the LCAT reaction in reconstituted discoidal HDL particles, within large spherical CE containing particles, it may be limited in this function, and apoE may then play a key role. Indeed, earlier studies suggested a requirement for apoE and LCAT in promoting significant HDL CE formation and cholesterol efflux from macrophages (31) and a structural role of apoE in enabling swine HDL to incorporate additional layers of CE within its core (32). These findings are also consistent with recent observations of Parks and colleagues who found 

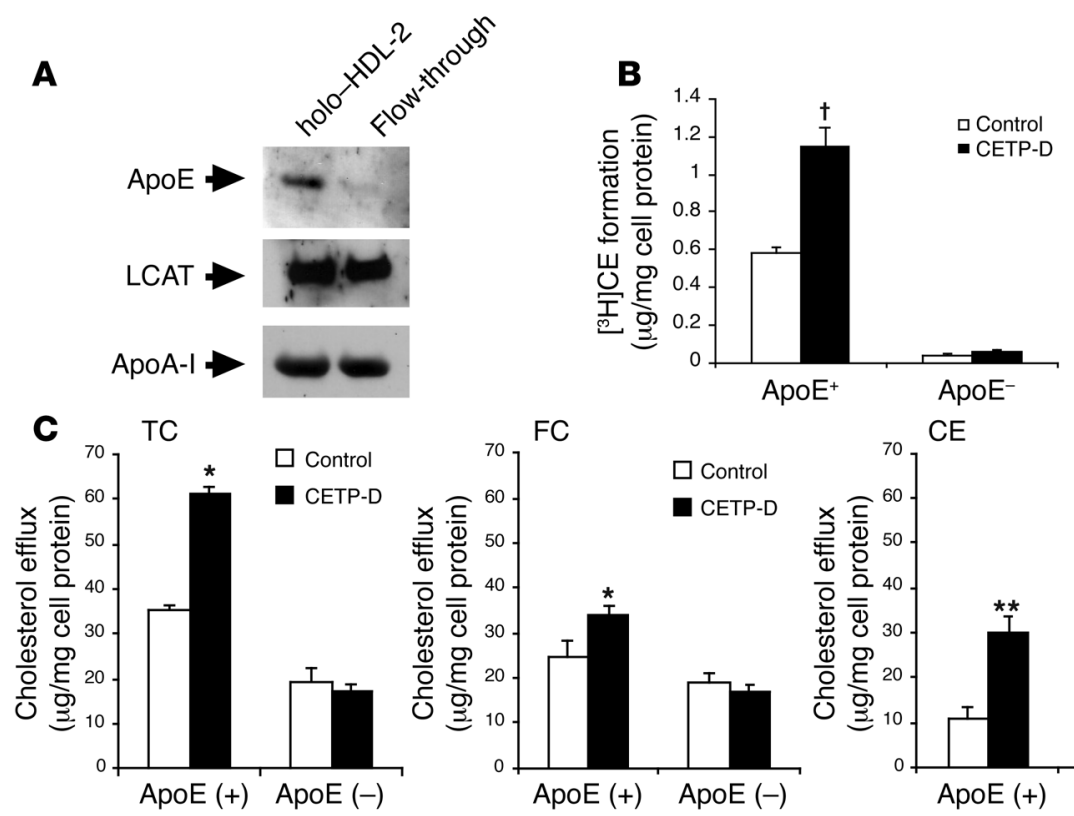

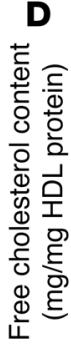
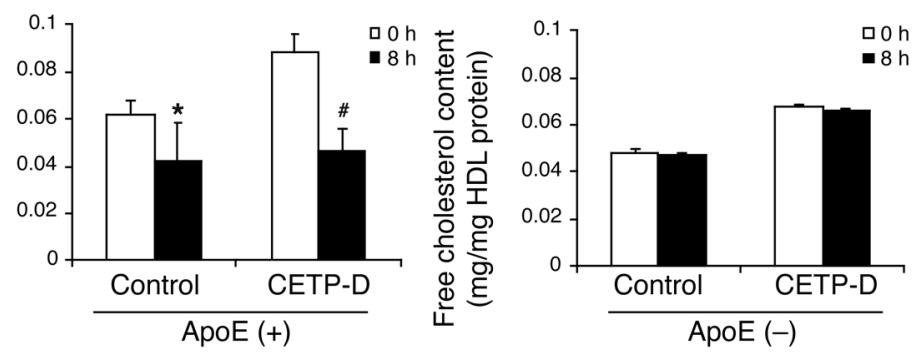

Figure 5

Net cholesterol efflux to apoE-free HDL-2 from macrophages and CE formation in media containing apoE-free HDL-2. ApoE was removed from HDL by heparin-sepharose chromatography, then the cholesterol efflux studies were using the holo-HDL-2 fraction or HDL-2 depleted of apoE. (A) Immunoblot analysis of LCAT, apoE, and apoA-I in the holo-HDL-2 fraction and the apoE-free HDL-2. (B) Isotopic CE formation in media containing holo-HDL-2 or apoE-free HDL-2. THP-1 macrophages were incubated in RPMI-1640 containing [ $\left.{ }^{3} \mathrm{H}\right]$ AcLDL with T0 for 24 hours, then cholesterol efflux was performed for 8 hours in RPMl-1640 containing HDL-2 (50 $\mu \mathrm{g} / \mathrm{ml}$ HDL protein). The data represent mean $\pm \mathrm{SD}$ of an experiment performed in triplicate. ${ }^{\dagger} P<0.001$, versus apoE ${ }^{+}$control HDL-2. (C) Net cholesterol efflux to holo-HDL-2- and apoE-free HDL-2 from THP-1 macrophages. Macrophages were treated with T0 and AcLDL for 24 hours, and then cholesterol efflux was performed for 8 hours in RPMI-1640 containing HDL-2 (50 $\mu \mathrm{g} / \mathrm{ml} \mathrm{HDL}$ protein). The data represent mean \pm SD of the experiment performed in triplicate. ${ }^{\star} P<0.05$, ${ }^{\star \star} P<0.01$, versus apoE ${ }^{+}$-control HDL-2. (D) LCAT activity of HDL-2 particles. FC content of HDL-2 fraction was measured using the enzymatic method before and after incubation in plastic tubes for 8 hours at $37^{\circ} \mathrm{C}$ with gentle shaking. The reduced FC mass of HDL-2 was estimated as the actual LCAT activity. The data represent mean \pm SD of an experiment performed in triplicate. ${ }^{*} P<0.05$, ${ }^{\#} P<0.005$ versus 0 hours.

that apoE has a essential role in stimulating cholesterol esterification on LDL particles and suggested the importance of apoE as an LCAT activator on large spherical lipoproteins (22).

Gene knockdown and overexpression of ABCG1 indicated an essential role in the ability of CETP-D HDL to stimulate increased cholesterol efflux. ABCG1 appears to act in the macrophage plasma membrane to increase the availability of cholesterol to HDL as well as other lipoprotein and non-lipoprotein acceptors (5). However, ABCG1 does not specifically bind HDL, and the mechanism of efflux could be increased passive diffusion subsequent to an increase in plasma membrane cholesterol content. Our present findings suggest a somewhat limited capacity for ABCG1 to promote mass choles- terol efflux to normal apoE-free HDL. This could be because in the absence of cholesterol esterification, the HDL FC content rapidly reaches an equilibrium with the plasma membrane FC. However, in the presence of ongoing cholesterol esterification, the gradient between cells and HDL is continually restored, driving net cholesterol efflux.

Overall, our findings tend to refute the rather prevalent idea that HDL from subjects with CETP-D is dysfunctional (33). Moreover, SR-BI that has a key role in clearing HDL CE in the liver (at least in rodents) also efficiently promotes selective uptake from CETP-D $\operatorname{HDL}(34,35)$. In the absence of CETP, hepatic clearance of HDL CE presumably occurs by apoE-mediated particle uptake or via SR-BI. Thus, there is unlikely to be an overall defect in reverse cholesterol transport in CETP-D. However, the relative importance of different efflux pathways involving ABCG1, ABCA1, and SR-B1 in vivo is still unknown, and the possibility remains that a theoretically impaired ability of CETP-D to regenerate lipidpoor apoA-I could contribute to a defect in cholesterol efflux via ABCA1.

Recent studies using CETP inhibitors in rabbits have provided encouraging results and suggest that HDL elevation is antiatherogenic $(12,13$, $36,37)$. However, human clinical data on atherosclerotic cardiovascular disease in subjects with genetic CETP-D has not led to any definite conclusion (38-41). The final verdict on CETP inhibition as a therapy for atherosclerotic cardiovascular disease will come from ongoing phase III trials.

\section{Methods}

Study subjects. The subjects were 4 Japanese patients referred to Osaka University Hospital, Japan, who were diagnosed as homozygous CETP-D due to a G-to-A mutation in the $5^{\prime}$-splice donor site of intron 14 (42); informed consent was obtained from each patient. Four normolipidemic healthy Japanese volunteers were used as controls.

Serum lipid and lipoprotein analysis. Fasting serum levels of TC, TG, and HDL cholesterol were assayed by standard enzymatic procedures. The serum concentrations of apoA-I, apoB, and apoE were determined by a single radial immunodiffusion (43). CETP activity and protein mass of the patients were measured by the methods of Kato et al. (44) and Sato et al. (45).

Isolation of HDL from plasma. From all subjects, 12-hour fasting venous blood samples were collected into syringes containing $0.01 \% \mathrm{Na}_{2}$ EDTA. HDL-2 and HDL-3 fractions were isolated from plasma by sequential ultracentrifugation at densities of 1.125 and $1.210 \mathrm{~g} / \mathrm{ml}$, respectively. 
The isolated lipoprotein fractions were dialyzed against PBS, pH 7.4, using cellulose ester dialysis membrane (mol wt cutoff: $10 \mathrm{kDa}$; Spectrum Laboratories Inc.). Cholesterol, TG, and phospholipid concentrations in HDL-2 and HDL-3 were determined using commercial kits (Wako Pure Chemical Industries Ltd.). The diameter of HDL particles was determined by electrophoresis on $4-15 \%$ nondenaturing polyacrylamide gradient gels (Bio-Rad). Electrophoresis was performed in Trisborate buffer (0.09 M Tris, 0.09 M boric acid, 0.002 $\mathrm{M} \mathrm{Na}_{2}$ EDTA, $\mathrm{pH}$ 8.3), and gels were stained with Coomassie blue.

Cells. HEK 293 cells and thioglycollate-elicited mouse (wild-type mice: 8-week-old C57BL/6 male mice from the Jackson Laboratory) peritoneal macrophages were grown in DMEM containing 10\% FBS. Human monocyte cell line THP-1 cells were grown in growth medium (RPMI-1640 medium containing $10 \%$ inactive FBS with $50 \mu \mathrm{M} \beta$-mercaptoethanol) and were differentiated to macrophages in growth medium containing $200 \mathrm{ng} / \mathrm{ml}$ phorbol 12-myristate 13-acetate (PMA; Sigma-Aldrich).

Plasmid constructs and cell transfection. The plasmid constructs expressing mouse ABCG1 were prepared by cloning mouse full-length cDNAs into pCMV-SPORT6 vector. When HEK 293 cells reached $70-80 \%$ confluence in collagen-coated plates, the cells were transiently transfected with the constructs using LipofectAMINE 2000 (Invitrogen Corp.). Twenty-four hours after the transfection, the cells were used for cholesterol efflux experiments.

Net cholesterol efflux assays. Twenty-four hours after HEK 293 cells were transfected with plasmid expressing ABCG1 or control empty vector (mock), the cells were incubated for 8 hours in DMEM containing $0.1 \%$ BSA in the presence or absence of HDL-2 $(50 \mu \mathrm{g}$ protein $/ \mathrm{ml})$. After the incubation with HDL, the lipid fractions were extracted from the efflux medium with hexane. After drying under $\mathrm{N}_{2}$ gas, the mass of cholesterol dissolved in the hexane was determined by using gas-liquid chromatography.

Thioglycollate-elicited mouse peritoneal macrophages or THP-1 macrophages were first incubated in DMEM or RPMI-1640 containing 0.5\% FBS with or without LXR agonist (T0, $3 \mu \mathrm{M}$; Sigma-Aldrich) and with AcLDL $(50 \mu \mathrm{g}$ protein $/ \mathrm{ml})$ for 24 hours. Then cholesterol efflux was performed for 8 hours at $37^{\circ} \mathrm{C}$ in DMEM or RPMI-1640/0.1\% BSA with the indicated concentrations of HDL-2 or HDL-3. In each experiment, medium containing HDL was incubated in wells either with cells or without cells. At the end of the 8-hour incubation, the lipid fractions were extracted from the efflux medium or cells using hexane and hexane/isopropanol (3:2, vol/vol), respectively. The HDL-mediated net cholesterol efflux was calculated by subtraction of cholesterol mass of the medium cultured without macrophages from that of the medium cultured with cells. The percentage of cholesterol efflux to HDL from cells was calculated by HDL-mediated net cholesterol efflux in media divided by total cellular cholesterol mass. In selected experiments, the reduction in cholesterol mass in cells (FC and $\mathrm{CE}$ ) was also determined by subtracting the cholesterol mass for cells incubated in media without HDL from that of cells incubated in media with HDL. When measured in the same experiment, the reduction of cholesterol mass in cells approximated the increase in cholesterol mass in media.

Isotopic cholesterol efflux assay. Mouse macrophages were incubated in DMEM containing $\left[{ }^{3} \mathrm{H}\right]$ AcLDL $(50 \mu \mathrm{g} / \mathrm{ml})$ with T0 $(3 \mu \mathrm{M})$ for 24 hours. Cholesterol efflux was performed for 2 or 5 hours in DMEM/0.1\% BSA containing HDL-2 or HDL-3 $(50 \mu \mathrm{g} / \mathrm{ml}$ HDL protein). The cholesterol efflux is expressed as the percentage of the radioactivity released from the cells in the medium relative to the total radioactivity in cells plus medium.

siRNA-mediated macrophage RNA interference. RNA interference to suppress ABCG1 expression in mouse macrophages was performed as previously described (5). For cholesterol efflux assays, mouse macrophages were cultured in DMEM $/ 10 \% \mathrm{FBS}$ at $37^{\circ} \mathrm{C}$ for 24 hours. The culture medium was removed and changed to DMEM/0.5\% FBS. The cells were transfected with siRNA (160 nM) and LipofectAMINE 2000 for 48 hours. Twenty-four hours after the transfection, T0 $(3 \mu \mathrm{M})$ and AcLDL $(50 \mu \mathrm{g}$ protein $/ \mathrm{ml})$ were added to each well. The cells were washed with PBS, and then the cholesterol efflux was performed for 8 hours in DMEM/0.1\% BSA in the presence or absence of HDL-2 (50 $\mu \mathrm{g} / \mathrm{ml}$ HDL protein). Protein levels of ABCG1 normalized against $\beta$-actin were determined by Western blot analysis.

Immunoblot analysis of LCAT, apo A-1, and apoE in HDL. The protein content of the HDL separated from plasma was determined by the Bio-Rad protein assay. HDL samples $(20 \mu \mathrm{g})$ were boiled with SDS sample buffer $(0.00625 \mathrm{M}$ Tris- $\mathrm{HCl}$ pH6.8, 2\% SDS, 5\% 2-mercaptoethanol, 10\% sucrose, and $0.002 \%$ Coomassie blue R-250) at $95^{\circ} \mathrm{C}$ for 10 minutes. Equal amounts of $\mathrm{HDL}$ protein were separated by SDS- PAGE on $4-15 \%$ SDS-polyacrylamide gradient gel (Bio-Rad) and then blotted onto nitrocellulose membrane. LCAT, apoA-I, and apoE proteins were detected using anti-human LCAT polyclonal antibody (400-107A2; Novus Biological Inc.), anti-human apoA-I monoclonal antibody (ab17278; Abcam), or anti-human apoE monoclonal antibody (ab1906; Abcam). Primary antibodies were detected using a peroxidase-conjugated anti-rabbit or anti-mouse antibody and revealed by chemiluminescence (ECL Plus reagent; Amersham Biosciences).

Treatment of HDL with SMase. After HDL-2 was dialyzed against PBS, $\mathrm{pH}$ 7.4, without EDTA, the incubation of HDL-2 with SMase from Bacillus cereus (Sigma-Aldrich) was carried out for 1 hour at $37^{\circ} \mathrm{C}$ using $1.4 \mathrm{U}$ SMase/mg HDL protein. The reaction was stopped by the addition of $1 \mathrm{mM}$ EDTA. To remove free SMase from the samples, HDL-2 was dialyzed against PBS with EDTA, pH 7.4, using cellulose ester dialysis membrane (MWCO: 100K; Spectrum Laboratories Inc.).

Measurement of SM and PC content of HDL. Total phospholipid content of HDL was assayed by the enzymatic method (Wako Pure Chemical Industries Ltd.). SM content of HDL was measured as previously described (46). PC concentration was obtained by subtracting the concentration of SM from that of the total phospholipid.

HDL association with T0-stimulated mouse peritoneal macrophages. HDL-2 was labeled with $\left.{ }^{125} \mathrm{I}\right]$ in IODO-GEN Pre-Coated Iodination Tubes (Pierce Biotechnology), and then free- $\left[{ }^{125} \mathrm{I}\right]$ was removed from the labeled HDL-2 samples using NAP5 columns (Amersham Biosciences), and $\left[{ }^{125} \mathrm{I}\right] \mathrm{HDL}-2$ was dialyzed in PBS, pH7.4, for 24 hours. For HDL-2cell association, T0-stimulated mouse macrophages were incubated with $\left[{ }^{125} \mathrm{I}\right] \mathrm{HDL}-2(1.5 \mu \mathrm{g} / \mathrm{ml})$ in DMEM/0.1\% BSA with or without heparin $\left(5 \mathrm{mg} / \mathrm{ml}\right.$; Sigma-Aldrich) for 1 hour at $37^{\circ} \mathrm{C}$. After washing 3 times with fresh medium, the cells were lysed with $0.1 \% \mathrm{SDS} / 0.1 \mathrm{~N} \mathrm{NaOH}$ lysis buffer, and radioactivity was determined by $\gamma$ counting. Nonspecific binding was determined by the addition of 50-fold excess of unlabeled HDL-2. Specific cell association was calculated by the subtraction of nonspecific cell association from total cell association.

Isotopic CE formation by HDL-2. HDL-2 was pretreated with LCAT inhibitor, E600 ( $2 \mathrm{mM}$; Sigma-Aldrich) for 0.5 hours at room temperature. THP-1 macrophages were incubated in RPMI-1640 containing $\left[{ }^{3} \mathrm{H}\right] \mathrm{AcLDL}$ $(50 \mu \mathrm{g} / \mathrm{ml})$ with T0 $(3 \mu \mathrm{M})$ for 24 hours, and cells were washed with fresh medium. Cholesterol efflux was performed for 8 hours in RPMI-1640/0.1\% BSA containing $200 \mathrm{ng} / \mathrm{ml}$ of PMA in the presence of HDL-2 $(50 \mu \mathrm{g} / \mathrm{ml}$ HDL protein) pretreated with or without E600. HDL-2 pretreated with E600 was directly added to the efflux medium (final concentration of E600 in the media was $0.2 \mathrm{mM}$ ). Lipids extracted from media with hexane were applied to thin-layer chromatography (TLC) plates $(20 \times 20 \mathrm{~cm}$; Silica Gel 60; Merck KGaA). Hexane/ethyl ether/acetic acid (70:30:1, vol/ $\mathrm{vol} / \mathrm{vol}$ ) was used to develop the TLC plates. Cholesteryl oleate was used as a nonradioactive $\mathrm{CE}$ standard. The location of $\mathrm{CE}$ for each sample was determined by visualization under iodine. $\left[{ }^{3} \mathrm{H}\right] \mathrm{CE}$ mass was measured by liquid scintillation counting.

Statistics. Statistical significance was determined using 2-tailed Student's $t$ test. $P<0.05$ was considered statistically significant. 


\section{Acknowledgments}

The authors would like to thank Shizuya Yamashita (Osaka University) for providing serum from CETP-deficient patients. This study was supported by grants from the NIH (HL22682), the Mochida Memorial Foundation for Medical and Pharmaceutical Research, and the Kanae Foundation for Life \& SocioMedical Science.

1. Glomset, J.A. 1968. The plasma lecithin:cholesterol acyltransferase reaction. J. Lipid Res. 9:155-167.

2. Ji, Y., et al. 1997. Scavenger receptor BI promotes high density lipoprotein-mediated cellular cholesterol efflux. J. Biol. Chem. 272:20982-20985.

3. Wang, N., Silver, D.L., Thiele, C., and Tall, A.R. 2001. ATP-binding cassette transporter A1 (ABCA1) functions as a cholesterol efflux regulatory protein. J. Biol. Chem. 276:23742-23747.

4. Hara, H., and Yokoyama, S. 1992. Role of apolipoproteins in cholesterol efflux from macrophages to lipid microemulsion: proposal of a putative model for pre-beta high density lipoprotein pathway. Biochemistry. 31:2040-2046.

5. Wang, N., Lan, D., Chen, W., Mastuura, F., and Tall, A.R. 2004. ATP-binding cassette transporters G1 and G4 mediate cellular cholesterol efflux to highdensity lipoproteins. Proc. Natl. Acad. Sci. U. S. A. 101:9774-9779.

6. Kennedy, M.A., et al. 2005. ABCG1 has a critical role in mediating cholesterol efflux to HDL and preventing cellular lipid accumulation. Cell Metab. 2:121-131.

7. Kennedy, M.A., et al. 2001. Characterization of the human ABCG1 gene: liver X receptor activates an internal promoter that produces a novel transcript encoding an alternative form of the protein. J. Biol. Chem. 276:39438-39447.

8. Costet, P., Luo, Y., Wang, N., and Tall, A.R. 2000. Sterol-dependent transactivation of the $\mathrm{ABC} 1$ promoter by the liver $\mathrm{X}$ receptor/retinoid $\mathrm{X}$ receptor. J. Biol. Chem. 275:28240-28245.

9. Inazu, A., et al. 1994. Genetic cholesteryl ester transfer protein deficiency caused by two prevalent mutations as a major determinant of increased levels of high density lipoprotein cholesterol. J. Clin. Invest. 94:1872-1882.

10. Brown, M.L., et al. 1989. Molecular basis of lipid transfer protein deficiency in a family with increased high-density lipoproteins. Nature. 342:448-451.

11. Inazu, A., et al. 1990. Increased high-density lipoprotein levels caused by a common cholesterylester transfer protein gene mutation. N. Engl. J. Med. 323:1234-1238.

12. Brousseau, M.E., et al. 2004. Effects of an inhibitor of cholesteryl ester transfer protein on HDL cholesterol. N. Engl. J. Med. 350:1505-1515.

13. Clark, R.W., et al. 2004. Raising high-density lipoprotein in humans through inhibition of cholesteryl ester transfer protein: an initial multidose study of torcetrapib. Arterioscler. Thromb. Vasc. Biol. 24:490-497.

14. De Grooth, G.J., et al. 2002. Efficacy and safety of a novel cholesteryl ester transfer protein inhibitor, JTT-705, in humans: a randomized phase II doseresponse study. Circulation. 105:2159-2165.

15. Brewer, H.B., Jr. 2004. Increasing HDL cholesterol levels. N. Engl. J. Med. 350:1505-1515.

16. Rye, K.A., and Barter, P.J. 2004. Formation and metabolism of prebeta-migrating, lipid-poor apolipoprotein A-I. Arterioscler. Thromb. Vasc. Biol. 24:421-428

17. Ishigami, M., et al. 1994. Large and cholesteryl ester-rich high density lipoproteins in cholesteryl ester transfer protein (CETP) deficiency can not
Received for publication December 7, 2005, and accepted in revised form February 27, 2006.

Address correspondence to: Fumihiko Matsuura, Department of Cardiovascular Medicine, B5, Osaka University, 2-2 Yamadaoka, Suita, Osaka 565-0871, Japan. Phone: 81-6-6879-3732; Fax: 81-66879-3739; E-mail: fc-mastu@fb4.so-net.ne.jp. protect macrophages from cholesterol accumulation induced by acetylated low-density lipoproteins. J. Biochem. (Tokyo). 116:257-262.

18. Yamashita, S., et al. 1991. Characterization of plasma lipoproteins in patients heterozygous for human plasma cholesteryl ester transfer protein (CETP) deficiency: plasma CETP regulates highdensity lipoprotein concentration and composition. Metabolism. 40:756-763.

19. Yamashita, S., et al. 1990. Accumulation of apolipoprotein E-rich high density lipoproteins in hyperalphalipoproteinemic human subjects with plasma cholesteryl ester transfer protein deficiency. J. Clin. Invest. 86:688-695.

20. Fielding, C.J., and Fielding, P.E. 1981. Evidence for a lipoprotein carrier in human plasma catalyzing sterol efflux from cultured fibroblasts and its relationship to lecithin:cholesterol acyltransferase. Proc. Natl. Acad. Sci. U. S. A. 78:3911-3914.

21. Francone, O.L., Haghpassand, M., Bennett, J.A., Royer, L., and McNeish, J. 1997. Expression of human lecithin:cholesterol acyltransferase in transgenic mice: effects on cholesterol efflux, esterification, and transport. J. Lipid Res. 38:813-822.

22. Zhao, Y., Thorngate, F.E., Weisgraber, K.H., Williams, D.L., and Parks, J.S. 2005. Apolipoprotein E is the major physiological activator of lecithin-cholesterol acyltransferase (LCAT) on apolipoprotein B lipoproteins. Biochemistry. 44:1013-1025.

23. Bolin, D.J., and Jonas, A. 1996. Sphingomyelin inhibits the lecithin-cholesterol acyltransferase reaction with reconstituted high density lipoproteins by decreasing enzyme binding. J. Biol. Chem. 271:19152-19158.

24. Subbaiah, P.V., and Liu, M. 1993. Role of sphingomyelin in the regulation of cholesterol esterification in the plasma lipoproteins. J Biol. Chem. 268:20156-20163.

25. Innerarity, T.L., Pitas, R.E., and Mahley, R.W. 1982. Modulating effects of canine high density lipoproteins on cholesteryl ester synthesis induced by beta-very low density lipoproteins in macrophages. Possible in vitro correlates with atherosclerosis. Arteriosclerosis. 2:114-124.

26. Asztalos, B.F., et al. 2005. Value of high-density lipoprotein (HDL) subpopulations in predicting recurrent cardiovascular events in the Veterans Affairs HDL Intervention Trial. Arterioscler. Thromb. Vasc. Biol. 25:2185-2191.

27. McPherson, R., Lau, P., Kussie, P., Barrett, H., and Tall, A.R. 1997. Plasma kinetics of cholesteryl ester transfer protein in the rabbit. Effects of dietary cholesterol. Arterioscler. Thromb. Vasc. Biol. 17:203-210.

28. Huang, Y., von Eckardstein, A., Wu, S., and Assmann, G. 1995. Cholesterol efflux, cholesterol esterification, and cholesteryl ester transfer by LpA-I and LpA-I/A-II in native plasma. Arterioscler. Thromb. Vasc. Biol. 15:1412-1418.

29. Tall, A.R., Abreu, E., and Shuman, J. 1983. Separation of a plasma phospholipid transfer protein from cholesterol ester/phospholipid exchange protein. J. Biol. Chem. 258:2174-2180.

30. Jiang, X.C., et al. 1999. Targeted mutation of plasma phospholipid transfer protein gene markedly reduces high-density lipoprotein levels. J. Clin.
Invest. 103:907-914

31. Gordon, V., Innerarity, T.L., and Mahley, R.W. 1983. Formation of cholesterol- and apoprotein Eenriched high density lipoproteins in vitro. J. Biol. Chem 258:6202-6212.

32. Tall, A.R., Atkinson, D., Small, D.M., and Mahley, R.W. 1977. Characterization of the lipoproteins of atherosclerotic swine. J. Biol. Chem. 252:7288-7293.

33. Ashen, M.D., and Blumenthal, R.S. 2005. Clinical practice. Low HDL cholesterol levels. N. Engl. J. Med. 353:1252-1260

34. Acton, S., et al. 1996. Identification of scavenger receptor SR-BI as a high density lipoprotein receptor. Science. 271:518-520.

35. Kinoshita, M., et al. 2004. Scavenger receptor type BI potentiates reverse cholesterol transport system by removing cholesterol ester from HDL. Atherosclerosis. 173:197-202.

36. Okamoto, H., et al. 2000. A cholesteryl ester transfer protein inhibitor attenuates atherosclerosis in rabbits. Nature. 406:203-207.

37. Shimoji, E., Zhang, B., Fan, P., and Saku, K. 2004. Inhibition of cholesteryl ester transfer protein increases serum apolipoprotein (apo) A-I levels by increasing the synthesis of apo A-I in rabbits. Atherosclerosis. 172:247-257

38. Hirano, K., et al. 1995. Atherosclerotic disease in marked hyperalphalipoproteinemia. Combined reduction of cholesteryl ester transfer protein and hepatic triglyceride lipase. Arterioscler. Thromb. Vasc. Biol. 15:1849-1856.

39. Hirano, K., et al. 1997. Genetic cholesteryl ester transfer protein deficiency is extremely frequent in the Omagari area of Japan. Marked hyperalphalipoproteinemia caused by CETP gene mutation is not associated with longevity. Arterioscler. Thromb. Vasc. Biol. 17:1053-1059.

40. Moriyama, Y., et al. 1998. A low prevalence of coronary heart disease among subjects with increased high-density lipoprotein cholesterol levels including those with plasma cholesteryl ester transfer protein deficiency. Prev. Med. 27:659-667.

41. Curb, J.D., et al. 2004. A prospective study of HDL$\mathrm{C}$ and cholesteryl ester transfer protein gene mutations and the risk of coronary heart disease in the elderly. J. Lipid Res. 45:948-953.

42. Brown, M., et al. 1989. Molecular basis of lipid transfer protein deficiency in a family with increased high-density lipoproteins. Nature. 342:448-451.

43. Goto, Y., Akanuma, Y., and Harano, Y. 1986. Determination by the SRID method of normal values of serum apolipoproteins (A-I, A-II, B, C-II, C-III, and E) in normallipidemic healthy Japanese subjects. J. Clin. Biochem. Nutr. 1:67-108.

44. Kato, H., Nakanishi, T., Arai, H., Nishida, H.I., and Nishida, T. 1989. Purification, microheterogenity and stability of human lipid transfer protein. J. Biol. Chem. 264:4082-4087.

45. Sato, T., et al. 1995. Enzyme-linked immunosorbent assay for cholesteryl ester transfer protein in human serum. Clin. Chim. Acta. 240:1-9.

46. Jiang, X.C., et al. 2000. Plasma sphingomyelin level as a risk factor for coronary artery disease. Arterioscler. Thromb. Vasc. Biol. 20:2614-2618. 